\title{
Doença de Ménière e vertigem postural
}

\author{
Mirella Boaglio ${ }^{1}$, Letícia Clemente Alvim Soares ${ }^{2}$, \\ Carmem Silvia Marsiglia Natal Ibrahim, \\ Fernando Freitas Ganança', \\ Oswaldo Laércio Mendonça Cruz
}

\section{Ménière's disease and positional vertigo}

Resumo / Summary Palavras-chave: Doença Ménière, vertigem postural
Key words: Ménière disease, vertigo
$\mathbf{O}$ bjetivo: A associação entre doença de Ménière e a vertigem postural tem sido descrita por vários autores. O objetivo deste estudo foi avaliar a presença do nistagmo de posicionamento e da sensação vertiginosa posicional em pacientes portadores de Doença de Ménière. Forma de estudo: Clínico prospectivo. Método: Foram avaliados 44 pacientes com diagnóstico definitivo de doença de Ménière segundo avaliação clínica e audiológica (preenchendo os critérios diagnósticos da American Academy of Otolaryngology) e confirmação da hidropisia endolinfática por eletrococleografia (relação SP/AP $>30 \%$ ). O estudo do nistagmo de posicionamento foi realizado por meio da manobra de Brandt-Daroff com utilização das lentes de Frenzel -13 dioptrias. O exame foi considerado alterado pela presença de nistagmo e/ou vertigem. Resultados: Na pesquisa do nistagmo de posicionamento, 7 pacientes (16\%) apresentaram nistagmo e vertigem, $23(52,2 \%)$ apresentaram vertigem sem o registro de nistagmo, e $14(31,8 \%)$ não apresentaram alteração alguma. Considerando a presença isolada de vertigem como alteração do exame e a presença de vertigem postural associada à presença de nistagmo postural, o total de alterações foi de 68,1\% (30 pacientes) nesta amostra. Conclusão: A associação entre Doença de Menière e vertigem postural é relevante e sua real incidência dependerá dos critérios utilizados para definir esta associação. Neste trabalho, tanto o desencadeamento de nistagmo quanto de sensação vertiginosa à manobra de Brandt-Daroff foram considerados positivos para a constatação desta associação. Com esta metodologia, a presença de vertigem postural em pacientes com Ménière foi de $68 \%$.
A

im: Several authors have described the association between Ménière disease and positional vertigo. The goal of the present study was to evaluate the presence of positional nystagmus and positional dizziness sensation in patients with Menière disease. Stud design: Clinical prospective. Method: 44 patients with definite diagnosis of Menière disease according to clinical and audiologic evaluations (meeting the diagnosis criteria of the American Academy of Otolaryngology) and the confirmation of endolymphatic hydrops by electrocochleography (SP/ $\mathrm{AP}>30 \%$ ). The research of positional nystagmus was elicited through Brandt-Daroff's maneuver. This examination was considered positive by the presence of the nystagmus and/or dizziness. Results: During the investigation of positional nystagmus, 7 patients (16\%) presented nystagmus and dizziness, 23 patients (52.2\%) presented dizziness without nystagmus, and the total number of abnormalities was $68.1 \%$ (30 patients) in this sample. Conclusion: The association between Menière disease and positional vertigo is relevant and its real occurrence will depend on the criteria used in order to define this association. In this paper, both triggering of the nystagmus and the dizzy sensation at Brandt-Daroff's maneuver were considered positive to define the association. The presence of positional vertigo in patients with Menière disease using this methodology was $68 \%$.

\footnotetext{
${ }^{1}$ Fonoaudióloga mestre pela Disciplina de Otorrinolaringologia/Ciências Otorrinolaringológicas UNIFESP-EPM. ${ }^{2}$ Médica otorrinolaringologista mestranda pela Disciplina de Otorrinolaringologia e Cirurgia de Cabeça e Pescoço UNIFESP-EPM.

${ }^{3}$ Fonoaudióloga mestranda pela Disciplina de Otorrinolaringologia/Ciências Otorrinolaringológicas UNIFESP-EPM. ${ }^{4}$ Professor afiliado da Disciplina de Otoneurologia da UNIFESP-EPM.

${ }^{5}$ Professor convidado da Disciplina de Otorrinolaringologia Pediátrica da UNIFESP-EPM.

Endereço para correspondência: Letícia Clemente A. Soares - Av. 11 de junho 1006/63 - Vila Clementino 04041-003 São Paulo SP. E-mail: leticiaclemente@ig.com.br

Artigo recebido em 23 de outubro de 2002. Artigo aceito em 17 de janeiro de 2003
} 


\section{INTRODUÇÃO}

A tríade sintomática composta por zumbido, perda auditiva e vertigem, em episódios paroxísticos, foi descrita por Prosper Ménière em 1861, junto à Academia Imperial de Medicina de Paris (Memoire sur des de l'oreille interne donnant lieu a des symptomes de congestion cerebrale apoplectiforme), a partir de um um grupo de pacientes diagnosticados como portadores de "congestão cerebral apoplectiforme"1. Pela primeira vez, sugeriu que o sistema auditivo era repentinamente afetado, com o surgimento de zumbido e diminuição da audição, e, sendo a orelha interna o local acometido, o aparecimento de vertigem, tontura e desequilibrio, acompanhados por náusea, vômito e síncope, poderia ser explicado sem que houvesse envolvimento do sistema nervoso central.

Em 1874, Charcot ${ }^{2}$ denominou tal alteração como "Doença de Ménière". Depois das descrições histopatológicas de Hallpike e Cairns em 1938³ a doença de Ménière é atualmente reconhecida como a expressão clínica de uma síndrome idiopática de hidropisia endolinfática.

A constatação definitiva da alteração fisiopatológica que caracteriza a doença só pode ser comprovada por estudo anatomopatológico de ossos temporais (post morten) e, portanto, o seu diagnóstico deve ser baseado em critérios clínicos bem definidos. Segundo o Comitê de Audição e Equilibrio da Academia Americana de Otorrinolaringologia ${ }^{4}$, em 1995, a presença de hidropisia endolinfática pode ser inferida a partir da ocorrência de episódios espontâneos e recorrentes de vertigem, com duração mínima de 20 minutos, acompanhados por náusea, ânsia e/ou vômito, sem perda da consciência, com nistagmo horizonto-rotatório sempre presente, associados à perda de audição, plenitude aural e zumbido, no lado afetado.

A vertigem postural paroxística benigna (VPPB) caracteriza-se por episódios paroxísticos de vertigens súbitas e fugazes que surgem quando a cabeça do paciente é colocada em uma determinada posição. É uma condição que pode aparecer isoladamente ou associar-se a outras doenças labirínticas, normalmente desencadeando e/ou exarcebando uma crise vertiginosa 5 . O diagnóstico da VPPB baseia-se na história clínica e no exame físico, uma vez que a vertigem e o nistagmo podem ser reproduzidos no consultório ao movimentar-se a cabeça do paciente para a posição de Hallpike. O nistagmo aparece sempre após um período de latência de aproximadamente 5 segundos, sendo normalmente rotatório ou horizonto-rotatório, fatigável e revertendo de direção quando o paciente reassume a posição ortostática, quando do acometimento dos canais semicirculares posteriores; sendo horizontal quando do acometimento dos canais semicirculares laterais; e sendo vertical e inferior quando do acometimento dos canais semicirculares superiores.

A vertigem postural associada à doença de Ménière já vem sendo descrita na literatura ${ }^{2,5,6,7,8,9}$. Paparella ${ }^{10}$, em
1984, já acreditava que a hidropisia na parte anterior do labirinto, ou seja, no ducto coclear e sáculo, era o achado fisiológico mais importante na doença de Ménière. Segundo este autor, na maioria dos casos havia uma ruptura na membrana de Reissner e o sáculo poderia se distender até os limites dos canais semi-circulares. O sáculo distendido serviria como reservatório de endolinfa e, esta distensão, por sua vez, poderia gerar a vertigem, inclusive a vertigem posicional. Outra possibilidade seria a ocorrência de alterações da composição da endolinfa, especialmente pela contaminação entre a endolinfa e a perilinfa, favorecendo a formação de sedimento endolinfático que determinaria o aparecimento da vertigem postural ${ }^{9,11,12}$

Além disso, a própria hidropsia poderia causar lesão na mácula utricular, causando deslocamento das otocônias.

\section{OBJETIVO}

Avaliar a presença de vertigem posicional em pacientes com doença de Ménière por meio da pesquisa de nistagmo de posicionamento.

\section{METODOLOGIA}

Foram avaliados 44 pacientes, sendo 35 do sexo feminino (idade entre 21 e 72 anos, com idade média de 46,7 anos) e 9 do masculino (idade entre 27 e 61 anos, com idade média de 45,2 anos) com idade média geral de 46,4 anos. O diagnóstico definitivo de Doença de Ménière foi realizado em período intercrítico, segundo os critérios clínicos e audiométricos elaborados no consenso de 1995 da Academia Americana de Otorrinolaringologia ${ }^{4}$ : dois ou mais episódios de vertigem com duração de 20 minutos ou mais, perda auditiva audiometricamente documentada em pelo menos uma ocasião, zumbidos ou sensação de pressão no ouvido afetado.

Todos os pacientes foram avaliados no Ambulatório de Otoneurologia da UNIFESP-EPM e submetidos a anamnese, otoscopia, audiometria tonal e vocal, imitanciometria e exame vestibular computadorizado ${ }^{13}$ (VEC-WIN/ Neurograff). Além do diagnóstico clínico e estadiamento da doença, a presença de hipertensão endolinfática foi comprovada pela eletrococleografia (Echog) cujo critério de inclusão foi uma relação SP/AP superior a 30\%. A pesquisa de nistagmo de posicionamento foi realizada por meio da manobra de Brandt-Daroff com utilização das lentes de Frenzel -13 dioptrias. O exame foi considerado alterado pela presença de nistagmo e/ou vertigem posicionais.

\section{RESULTADOS}

Dos 44 pacientes avaliados, 14 (31,8\%) não apresentaram alterações à prova de pesquisa do nistagmo de posicionamento e 30 (68,2\%) apresentaram alterações, 
Tabela 1. Prevalência de achados à pesquisa do nistagmo de posicionamento em pacientes com doença de Ménière segundo sexo.

\begin{tabular}{|c|c|c|c|c|c|c|}
\hline \multirow{2}{*}{$\begin{array}{c}\text { NISTAGMO DE } \\
\text { POSICIONAMENTO }\end{array}$} & \multicolumn{2}{|c|}{ MASCULINO } & \multicolumn{2}{|c|}{ FEMININO } & \multicolumn{2}{|c|}{ TOTAL } \\
\hline & $\mathbf{N}$ & $\%$ & $\mathbf{N}$ & $\%$ & $\mathbf{N}$ & $\%$ \\
\hline Sem alteração & 3 & $(33,3 \%)$ & 11 & $(31,4 \%)$ & 14 & $(31,8 \%)$ \\
\hline Vertigem & 5 & $(55,5 \%)$ & 18 & $(51,4 \%)$ & 23 & $(52,2 \%)$ \\
\hline Vertigem e nistagmo & 1 & $(11,2 \%)$ & 6 & $(17,2 \%)$ & 7 & $(16 \%)$ \\
\hline TOTAL & 9 & $(100 \%)$ & 35 & $(100 \%)$ & 44 & $(100 \%)$ \\
\hline
\end{tabular}

sendo $23(52,2 \%)$ apenas vertigem e $7(16 \%)$ vertigem e nistagmo. Dos pacientes com alteração da prova, 6 (20\%) eram do sexo masculino e 24 (80\%) do sexo feminino. Nenhuma diferença foi observada quanto a incidência entre vertigem e vertigem + nistagmo em relação ao sexo (Tabela 1).

\section{DISCUSSÃO}

A concomitância ou associação de VPPB com a doença de Ménière tem sido descrita por diversos autores. Hughes, Proctor $^{11}$ avaliaram 781 pacientes com queixa de vertigem postural. Desses, 151 (19,3\%) apresentaram nistagmo posicional e foram diagnosticados como portadores de VPPB. A presença de doenças coexistentes ou associadas à VPPB foi observada em 99 pacientes (65,6\%), sendo que em 45 $(45,4 \%)$ a associação era com a doença de Ménière. Observaram que a instalação da doença de Ménière precedia a VPPB na maioria das vezes. A origem periférica das duas doenças e a possível liberação de otocônias por lesão do utrículo pela hidropisia e hipertensão endolinfática seriam possíveis explicações para o fato.

Gross et al. ${ }^{9}$ avaliaram 9 pacientes com diagnóstico de doença de Ménière e VPPB concomitantes. Os sintomas da doença de Ménière eram anteriores ao aparecimento da $\mathrm{VPPB}$ em todos pacientes. Um paciente apresentava VPPB bilateral e Ménière unilateral. Os pacientes que apresentaram VPPB "intratável" foram submetidos a reposicionamento de otólitos com as manobras de Epley, sem sucesso satisfatório. A partir da análise desses dados, os autores hipotetizaram que alterações do balanço hídrico induzidas na mácula utrícular e sacular e uma eventual obstrução parcial do labirinto membranoso poderiam ser mecanismos envolvidos na coexistência das duas doenças.

Karlberg et al. ${ }^{12}$ avaliaram, por intermédio de estudo retrospectivo, 2847 pacientes com VPPB. Destes, 0,6\% $(n=16)$ sofriam de doença de Ménière, $0,8 \%(n=24)$ de vestibulopatia periférica aguda unilateral, $0,7 \%(n=21)$ de vestibulopatia periférica crônica bilateral, $0,4 \%(n=12)$ de vestibulopatia periférica crônica unilateral, $0,3 \%(n=8)$ de perda auditiva neurossensorial e o restante 97,2\% $(n=2766)$ apenas VPPB. No caso da doença de Ménière, aventaram a possibilidade da hidropisia endolinfática afetar o utrículo, resultando na perda das otocônias, provocando a VPPB secundária.

A alta prevalência de alterações encontradas na pesquisa do nistagmo de posicionamento, neste trabalho (prevalência de $68,1 \%$ de vertigem e/ou nistagmo posturais) são concordantes aos achados de Paparella ${ }^{10}$, Cruz, Cruz Filho ${ }^{7}$, Wexler et al. ${ }^{14}$, Haid et al. ${ }^{7}$, Mizukoshi et al. ${ }^{15}$ e Gross et al. ${ }^{9}$. Ressalta-se ainda que neste estudo não só a presença do nistagmo ocorrido isoladamente em $16 \%$ dos pacientes estudados foi valorizada, mas também a ocorrência de vertigem sem nistagmo, observada em $52,2 \%$ dos pacientes, considerada também como alteração na pesquisa do nistagmo de posicionamento.

\section{CONCLUSÕES}

- A ocorrência de alterações na pesquisa do nistagmo de posicionamento nos pacientes portadores de doença de Ménière estudados é relevante.

- Não houve diferenças significativas na prevalência de alterações na pesquisa do nistagmo de posicionamento em relação ao sexo.

\section{REFERÊNCIAS BIBLIOGRÁFICAS}

1. Meniérè P. Memoire sur des lesions de l'oreille interne donnant lieu a des symptomes de congestion cerebrale apoplectiforme. Gazette Medicale de Paris 1861,16:597-601.

2. Charcot JM. Conferences cliniques de Salpetriere: vertiges ab aure laesa (maladie de Ménière). Gazette des Hospiteaux Civils et Militaires 1874,47:73-4.

3. Hallpike C, Carins H. Observations on the pathology of Meniere's syndrome. J Laryngol Otol 1938;53:625.

4. Comitê em Audição e Equilíbrio da Academia Americana de Otorrinolaringologia.Guidelines for the diagnosis and evaluation of therapy in Ménière's disease. Otolaryng. Head Neck Surg 1995;113:181-85.

5. Ganança MM, Caovilla HH, Ganança FF, Ganança CF, Munhoz MSL, Silva MLG.Vertigem posicional paroxística benigna pós-doença de Ménière. In: Munhoz MSL, Ganança MM, Caovilla HH, Silva MLG Casos clínicos otoneurológicos típicos e atípicos. Rio de Janeiro: Atheneu; 2001. p.199-201. 
6. Friberg U, Stahle J, Svedberg, A. The natural course of Ménière's disease. Acta Otolaryngol (Stockh) 1984;406 Suppl:72-7.

7. Cruz NA, Cruz Filho NA. Doença de Ménière: parte III. F Med (BR) 1991b;103(3):129-36.

8. Haid CT, Watermeier D, Wolf SR, Berg M. Clinical survey of Ménière's disease. Acta Otolaryngol (Stockh) 1995;520 Suppl:25155.

9. Gross EM, Ress BD, Viirre ES, Nelson JR, Harris JP. Intractable benign paroxymal positional vertigo in patients with Ménière's disease. Larryngoscope 2000;110:655-659.

10. Paparella MM. Pathogenesis of Ménière's disease and Ménière's syndrome. Acta Otolaryngol (Stockh) 1984;406 Suppl:10-25.

11. Hughes CA, Proctor 1. Benign paroxysmal positional vertigo. Larryngoscope 1997;107:607-613.
12. Karlberg M, Hall K, Quickert N, Hinson J, Halmagyi M. What inner ear diseases cause benign paroxysmal positional vertigo? Acta Otolaryngol 2000;120:380-385.

13. Ganança CF, Souza JAC, Segantin LA, Caovilla HH, Ganança MM. Limites normais dos parâmetros de avaliação da Vectonistagmografia Digital Neurograff. Acta Awho 2000;19(2):105.

14. Wexler DB, Harker LA, Voots RJ, McCabe BF. Monothermal differential caloric testing in patients with Ménière's disease. Laryngoscope 1991;101:50-5.

15. Mizukoshi K, Watanabe Y, Shojaku H, Matsunaga T, Tokumasu K. Preliminary guidelines for reporting treatment results in Ménière's disease conducted by the Committee of the Japanese Society for Equilibrium Research 1993. Acta Otolaryngol (Stockh) 1995;519 Suppl:211-15. 